\title{
Parameter Optimization for Amplify-and-Forward Relaying Systems with Pilot Symbol Assisted Modulation Scheme
}

\author{
Yi WU, Matthias PÄTZOLD \\ Faculty of Engineering and Science, University of Agder, Grimstad, Norway \\ E-mail: \{yi.wu, matthias.paetzold\}@uia.no \\ Received March 7, 2009; revised March 16, 2009; accepted March 18, 2009
}

\begin{abstract}
Cooperative diversity is a promising technology for future wireless networks. In this paper, we consider a cooperative communication system operating in an amplify-and-forward (AF) mode with a pilot symbol assisted modulation (PSAM) scheme. It is assumed that a linear minimum mean square estimator (LMMSE) is used for the channel estimation at the receiver. A simple and easy-to-evaluate asymptotical upper bound (AUB) of the symbol-error-rate (SER) is derived for uncoded AF cooperative communication systems with quadrature amplitude modulation (QAM) constellations. Based on the AUB, we propose a criterion for the parameter optimization in the PSAM scheme. We discuss how the pilot spacing and the length of the Wiener filter should be chosen under the constraint of a tradeoff between pilot overhead, estimation accuracy, and receiver complexity. We also formulate an power allocation problem for the considered system. It is shown that the power allocation problem can optimally be solved by means of a gradient search method. Numerical simulations are presented to verify the correctness of the theoretical results and to demonstrate the benefits of the parameter optimization.
\end{abstract}

Keywords: Amplify-and-Forward, Cooperative Communication System, Imperfect Channel Estimations, Parameter Optimization

\section{Introduction}

Recently, a new form of spatial diversity called "cooperative diversity" has attracted much research interest because it provides effective diversity benefits for those devices that cannot be equipped with multiple antennas due to their size, complexity, and cost. The main idea behind cooperative diversity is that the mobile users in the neighborhood share the use of their antennas to assist each other with data transmission. Cooperative diversity is realized by collaboration among the users. If the channel fading from a user to the destination terminal is severe, then the information might be successfully transmitted through the cooperative users. Different cooperative protocols have been proposed to exploit the diversity that cooperative systems offer (see, e.g., [1-4] and the references therein). One commonly used protocol is the amplify-and-forward (AF) protocol, in which the relay terminal simply re-transmits a scaled version of the

This paper will be presented in part at the 69th IEEE Semiannual Vehicular Technology Conference, VTC2009-Spring, Barcelona, Spain, April 2009. received signal to the destination terminal. Depending on the scaling factor, the AF relaying scheme can be further divided into two types which are called fixed gain AF systems and variable gain AF systems [5].

The performance of AF cooperative systems has been studied in the past from different perspectives. For example, $[4,6,7]$ analyzed the performance of AF systems in terms of the outage probability and diversity gain under different assumptions for the amplifier gain. On the other hand, the authors of [8-12] derived exact expressions for the symbol-error-rate (SER) and presented various SER bounds for AF cooperative communication systems. However, all these papers have assumed that the perfect channel state information (CSI) is available at both the relay and destination terminal. More recently, [5] and [13] have studied the performance of AF cooperative communication systems with channel estimation errors by means of Monte Carlo simulations. The accurate SER expression for cooperative communication systems with a pilot symbol assisted modulation (PSAM) scheme employing a linear minimum mean square estimator (LMMSE) is derived in [14]. To the authors' best knowledge, no research has been conducted to solve the 
problem of parameter optimization and optimum power allocation for variable gain AF cooperative communication systems with a PSAM scheme.

The difficulty in optimizing the system design lies in the fact that the accurate SER expression is given in form of a double integral [14]. This prevents us from minimizing the SER directly. In this paper, we propose to use the asymptotic upper bound (AUB) of the SER to overcome these difficulties. In particular, we derive a tight expression for the AUB of the SER for the AF cooperative communication system with a PSAM scheme. The derived AUB has a simple form and no integrating operation is involved. Using the AUB of the SER, we present the criterion for the parameter choice in the PSAM scheme and show that two parameters used in this scheme, i.e., pilot spacing and Wiener filter length, can be chosen in a tradeoff between system performance, pilot overhead, and receiver complexity. With the derived tight $\mathrm{AUB}$, an optimum power allocation problem is also formulated for the AF cooperative communication system. Since the optimization of the power allocation is very complicated, as it is related to many terms, and obtaining an analytical solution is unlikely, we propose to find the optimum power allocation by means of a gradient search over a continuous range.

The rest of the paper is organized as follows. In Section 2, we describe the system model and some preliminaries of the AF cooperative system with a PSAM scheme. In Section 3, we derive an AUB of the SER for an AF cooperative communication system with LMMSE. In Section 4, we first deal with the parameter optimization for the PSAM scheme. Then, an optimum power allocation problem is formulated. A gradient search algorithm is also proposed to find the solution of the optimization problem. Various simulation results and their discussions are presented in Section 5. Finally, Section 6 contains the conclusions.

The following notation is used throughout the paper: $(\cdot)^{*},(\cdot)^{T},(\cdot)^{H}$, and $(\cdot)^{-1}$ denote the complex conjugate, vector (or matrix) transpose, conjugate transpose, and matrix inverse, respectively. The symbol $E[\cdot]$ denotes the expectation operator, $|z|$ represents the absolute value of a complex number $z$, and the complex Gaussian distribution with mean $m$ and covariance $P$ is denoted by $C \mathcal{N}(m, P)$.

\section{System Model}

We consider an AF cooperative communication system which consists of a source, relay, and destination terminal. The block diagram of the system is shown in Figure 1. We assume that each terminal is equipped with a single transmit and receive antenna and operates in a half-duplex mode, i.e., it cannot transmit and receive simultaneously. We adopt the so-called Protocol II proposed by Nabar et al. [6] as the user cooperative protocol. This means that two time slots are used to transmit one data symbol. The source terminal communicates with the relay and destination terminal during the first time slot. In the second time slot, only the relay terminal communicates with the destination terminal. This protocol realizes a maximum degree of broadcasting and exhibits no receive collisions [6]. To simplify the following analysis, we consider a symbol-by-symbol transmission, so that the time slot index 1 and 2 can be dropped. Throughout this paper, we assume that the system operates in a Rayleigh flat fading environment with perfect synchronization, and imperfect channel estimation is assumed at the receiver. As in [5], we use a PSAM scheme for the channel estimation. Pilot symbols are periodically inserted in data symbols with an insertion period of $L$ symbols. Since the design of an optimal channel estimator is very complex, we resort to a suboptimal LMMSE. We further assume that the data information symbols are equally probable over a constellation set composed of quadrature amplitude modulation (QAM) symbols of size $M$, and the pilot symbols are selected from a binary phase-shift keying (BPSK) constellation.

With these assumptions, let us look at the received signals corresponding to the $k$ th transmitted symbol. The received signals in the first time slot at the destination terminal and the relay terminal are given by

$$
\begin{aligned}
& r_{S D}(k)=\sqrt{P_{S}} h_{S D}(k) x(k)+n_{S D}(k) \\
& r_{S R}(k)=\sqrt{P_{S}} h_{S R}(k) x(k)+n_{S R}(k)
\end{aligned}
$$

respectively, where $P_{S}$ is the average power of the transmitted signal at the source terminal, $h_{S D}(k)$ and $h_{S R}(k)$ are the channel coefficients from the source terminal to the destination terminal with distribution $C \mathcal{N}\left(0, \sigma_{S D}^{2}\right)$ and from the source terminal to the relay terminal with distribution $C \mathcal{N}\left(0, \sigma_{S R}^{2}\right)$, respectively. The symbol $x(k)$ is the $k$ th transmitted symbol from the source terminal, and $n_{S D}(k)$ and $n_{S R}(k)$ are the additive receiver noises at the destination terminal and the relay terminal, respectively, with the same distribution $C \mathcal{N}\left(0, N_{0}\right)$. Throughout this paper, we assume

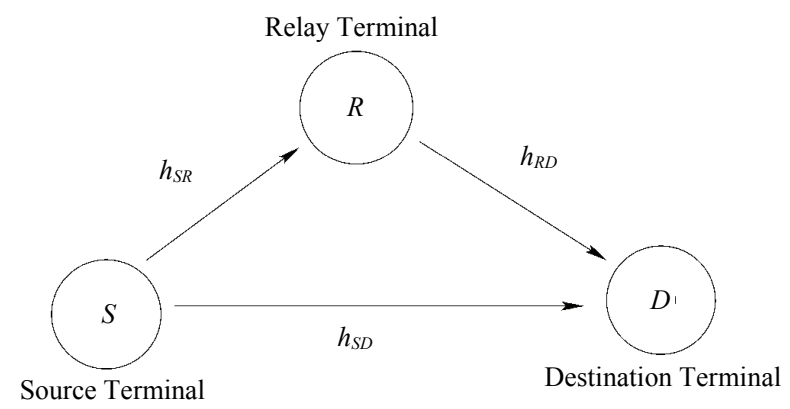

Figure 1. Block diagram of the AF cooperative communication system. 
that $E\left[|x(k)|^{2}\right]=1$, i.e., the transmitted symbols have an average energy of 1 . According to the Protocol II, the relay terminal will first normalize the received signal by a factor of $\sqrt{E\left(\left|r_{S R}(k)\right|^{2}\right)}$ (to ensure the unity of average energy). Then, the normalized signal will be amplified and forwarded to the destination terminal during the second time slot. Therefore, the received signal at the destination terminal within the second time slot is given by

$$
r_{R D}(k)=\frac{\sqrt{P_{R}}}{\sqrt{P_{S}\left|h_{S R}(k)\right|^{2}+N_{0}}} h_{R D}(k) r_{S R}(k)+n_{R D}(k)
$$

where $P_{R}$ is the average power of the transmitted signal at the relay terminal, $h_{R D}(k)$ is the channel coefficient from the relay terminal to the destination terminal with distribution $C \mathcal{N}\left(0, \sigma_{R D}^{2}\right)$, and $n_{R D}(k)$ is the additive receiver noise at the destination terminal with distribution $C \mathcal{N}\left(0, N_{0}\right)$. Using (2), we can rewrite (3) as

$$
r_{R D}(k)=\frac{\sqrt{P_{S} P_{R}}}{\sqrt{P_{S}\left|h_{S R}(k)\right|^{2}+N_{0}}} h_{S R}(k) h_{R D}(k) x(k)+n_{R D}^{\prime}(k)
$$

where

$$
n_{R D}^{\prime}(k)=\frac{\sqrt{P_{R}}}{\sqrt{P_{S}\left|h_{S R}(k)\right|^{2}+N_{0}}} h_{R D}(k) n_{S R}(k)+n_{R D}(k)
$$

Assuming that $n_{S R}(k)$ and $n_{R D}(k)$ are independent, it can be shown that the noise term $n_{R D}^{\prime}(k)$ is a complex Gaussian random variable with distribution $C \mathcal{N}\left(0,\left(\sqrt{P_{R}} / \sqrt{P_{S}\left|h_{S R}(k)\right|^{2}+N_{0}}+1\right) N_{0}\right)$. Since the PSAM scheme is used for the channel estimation, the packed transmission can be divided into blocks by pilot symbols. In each block, there are $L$ symbols in which the first time slot is assigned to a pilot symbol and the remaining $L-1$ symbols are assigned to data symbols. The channel estimation at each symbol position in a block is obtained using $N_{1}$ pilot symbols on the lefthand side of the symbol position and $N_{2}$ pilot symbols on the right-hand side of the symbol position. Therefore, $N=N_{1}+N_{2}$ pilot symbols are used to estimate the channel coefficient of the desired symbol position.

Let us denote the pilot symbols employed to estimate the channel gain $h_{S D}(k)$ of the desired data symbol $x(k)$ as an $N \times 1$ vector $\mathbf{p}_{S D}=\left[x\left(k-L\left(N_{1}-1\right)-l\right), \ldots\right.$, $\left.x(k-l), x(k+L-l), \ldots, x\left(k+L N_{2}-l\right)\right]^{T}$, where $l=1,2, \ldots$, $L-1$ is the offset of the desired data symbol $x(k)$ to the closest pilot symbol on its left side. Using (1), we obtain the received signal vector $\mathbf{r}_{S D}$, corresponding to the transmitted pilot vector $\mathbf{p}_{S D}$, at the destination terminal as

$$
\mathbf{r}_{S D}=\sqrt{P_{S}} \operatorname{diag}\left(\mathbf{p}_{S D}\right) \mathbf{h}_{S D}+\mathbf{n}_{S D}
$$

where $\mathbf{h}_{S D}=\left[h_{S D}\left(k-L\left(N_{1}-1\right)-l\right), \ldots, h_{S D}(k-l)\right.$,

$\left.h_{S D}(k+L-l), \ldots, h_{S D}\left(k+L N_{2}-l\right)\right]^{T}$ and

$\mathbf{n}_{S D}=\left[n_{S D}\left(k-L\left(N_{1}-1\right)-l\right), \ldots, n_{S D}(k-l)\right.$,

$\left.n_{S D}(k+L-l), \ldots, n_{S D}\left(k+L N_{2}-l\right)\right]^{T}$ are the channel coefficient and noise component at the pilot symbols' position for estimating $h_{S D}(k)$, respectively.

Without loss of generality, we assume that positive unit energy symbols are transmitted as pilot symbols, i.e., $\mathbf{p}_{S D}$ is an all-one vector. Then, (6) simplifies to

$$
\mathbf{r}_{S D}=\sqrt{P_{S}} \mathbf{h}_{S D}+\mathbf{n}_{S D}
$$

With these observations, the channel estimate for $h_{S D}(k)$ can be obtained by the LMMSE as [15]

$$
\hat{h}_{S D}(k)=\mathbf{w}_{S D} \mathbf{r}_{S D}
$$

where $\mathbf{w}_{S D}=\mathbf{c}_{h_{S D}, \mathbf{r}_{S D}}(l) \mathbf{C}_{\mathbf{r}_{S D}}^{-1}$ is an $1 \times N$ LMMSE filter vector, $\quad \mathbf{C}_{\mathbf{r}_{S D}}=E\left[\mathbf{r}_{S D} \mathbf{r}_{S D}^{H}\right]$ and $\mathbf{c}_{h_{S D}, \mathbf{r}_{S D}}(l)=$ $E\left[h_{S D}^{*}(k) \mathbf{r}_{S D}\right]$ are the autocorrelation matrix of $\mathbf{r}_{S D}$ and cross-correlation vector $h_{S D}(k)$ and $\mathbf{r}_{S D}$, respectively. From the LMMSE theory [15], we know that $\hat{h}_{S D}(k)$ is distributed as $C \mathcal{N}\left(0, \mathbf{c}_{h_{S D}}, \mathbf{r}_{S D}(l)\left(\mathbf{C}_{\mathbf{r}_{S D}}^{-1}\right)^{H}\right.$ $\left.\mathbf{c}_{h_{S D}, \mathbf{r}_{S D}}^{H}(l)\right)$. Let us define the discrete autocorrelation function of $h_{S D}(k)$ as $R_{S D}(\kappa)=E\left[h_{S D}(k) h_{S D}(k+\kappa)^{*}\right]$. Then, using the system model under consideration of the channel properties described above, we can finally express $\mathbf{C}_{\mathbf{r}_{S D}}$ and $\mathbf{c}_{h_{S D}, \mathbf{r}_{S D}}(l)$ as

$\mathbf{C}_{\mathbf{r}_{S D}}=\left[\begin{array}{cccc}P_{S} R_{S D}(0)+N_{0} & P_{S} R_{S D}(L) & \cdots & P_{S} R_{S D}((N-1) L) \\ P_{S} R_{S D}(L) & P_{S} R_{S D}(0)+N_{0} & \cdots & P_{S} R_{S D}((N-2) L) \\ \vdots & \vdots & \ddots & \vdots \\ P_{S} R_{S D}((N-1) L) & P_{S} R_{S D}((N-2) L) & \cdots & P_{S} R_{S D}(0)+N_{0}\end{array}\right]$

$$
\begin{aligned}
\mathbf{c}_{h_{S D}, \mathbf{r}_{S D}}(l)= & {\left[\sqrt{P_{S}} R_{S D}\left(-L\left(N_{1}-1\right)-l\right), \cdots, \sqrt{P_{S}} R_{S D}(L-l),\right.} \\
& \left.\cdots \sqrt{P_{S}} R_{S D}\left(L\left(N_{2}-1\right)-l\right)\right]
\end{aligned}
$$

From the LMMSE filter vector $\mathbf{w}_{S D}$, we can see that each data symbol position in a block requires a different estimator. However, due to the periodic pilot insertion, an identical estimator will be adopted at the same data symbol positions across all blocks in a packet. Therefore, without loss of generality, we will only consider $L-1$ 
different estimators for the data symbol positions in one particular block in the following analysis and employ the index $l$ instead of $k$ to distinguish them. With this in mind, we can express the estimation error of the $l$ th estimator as

$$
e_{S D}(l)=h_{S D}(l)-\hat{h}_{S D}(l)
$$

Furthermore, the estimation error $e_{S D}(l)$ is distributed as $C \mathcal{N}\left(0, \sigma_{e, S D}^{2}(l)\right)$, where $\left.\sigma_{e, S D}^{2}(l)\right)=\sigma_{S D}^{2}-\mathbf{c}_{h_{S D}, \mathbf{r}_{S D}}(l)$ $\left(\mathbf{C}_{\mathbf{r}_{S D}}^{-1}\right)^{H} \mathbf{c}_{h_{S D}}^{H}, \mathbf{r}_{S D}(l)$. From (11) it follows that we can model the channel gain $h_{S D}(l)$ as the sum of the channel estimate $\hat{h}_{S D}(l)$ and the estimation error $e_{S D}(l)$, i.e.,

$$
h_{S D}(l)=\hat{h}_{S D}(l)+e_{S D}(l)
$$

Similarly, we can model the channel gain from the source terminal to the relay terminal $h_{S R}(l)$ and the channel gain from the relay terminal to the source terminal $h_{R D}(l)$ as

$$
\begin{array}{r}
h_{S R}(l)=\hat{h}_{S R}(l)+e_{S R}(l) \\
h_{R D}(l)=\hat{h}_{R D}(l)+e_{R D}(l)
\end{array}
$$

where $\hat{h}_{S R}(l), e_{S R}(l), \hat{h}_{R D}(l)$, and $e_{R D}(l)$ can be attained using the same procedure as above.

\section{AUB Analysis for AF Cooperative Systems}

With the above assumption and the estimated channel gains, maximum ratio combining (MRC) [16] can be applied at the destination terminal to minimize the SER of the system. Define $B=1-1 / \sqrt{M}$ and $K_{Q}=3 /(M-1)$. The accurate SER expression for the considered system with M-QAM is [14]

$$
\bar{P}=\frac{1}{L-1} \sum_{l=1}^{L-1} P(l)
$$

where

$$
\begin{aligned}
& P(l)=\frac{1}{\pi} \int_{0}^{\pi / 2} \int_{0}^{1} \frac{4 B \eta(l)}{\alpha_{1}(l)+\frac{K_{Q}}{2 \sin ^{2} \theta}} f\left(l, x, \frac{K_{Q}}{2 \sin ^{2} \theta}\right) d x d \theta \\
& -\frac{1}{\pi} \int_{0}^{\pi / 4} \int_{0}^{1} \frac{4 B^{2} \eta(l)}{\alpha_{1}(l)+\frac{K_{Q}}{2 \sin ^{2} \theta}} f\left(l, x, \frac{K_{Q}}{2 \sin ^{2} \theta}\right) d x d \theta \\
& f(l, x, s)=\exp \left(-\frac{\alpha_{3}(l) \beta(l) x}{1-x}\right)[a(l, x, s)+b(l, x, s)] \\
& a(l, x, s)=\frac{\beta(l)}{\alpha_{2}(l)+\left(s-2 \alpha_{2}(l)+\alpha_{3}(l)\right) x+v(l, x, s)} \\
& v(l, x, s)=\left(-2 s+\alpha_{2}(l)-\alpha_{3}(l)\right) x^{2}+s x^{3}
\end{aligned}
$$

$$
\begin{aligned}
& b(l, x, s)=\frac{1}{\left(\alpha_{2}(l)+\left[s-\alpha_{2}(l)+\alpha_{3}(l)\right] x-s x^{2}\right)^{2}} \\
& \eta(l)=\alpha_{1}(l) \alpha_{2}(l) \alpha_{3}(l) \exp \left[\alpha_{3}(l) \beta(l)\right] \\
& \alpha_{1}(l)=\frac{\left[P_{S} \sigma_{e, S D}^{2}(l)+N_{0}\right]}{\left[P_{S} \sigma_{\hat{h}, S D}^{2}(l)\right]} \\
& \alpha_{2}(l)=\frac{\left(P_{R}+N_{0}\right) \sigma_{e, R D}^{2}(l)+N_{0}}{P_{R} \sigma_{\hat{h}, R D}^{2}(l)} \\
& \alpha_{3}(l)=\frac{P_{S} \sigma_{e, S R}^{2}(l)+N_{0}}{P_{S} \sigma_{\hat{h}, S R}^{2}(l)} \\
& \beta(l)=\frac{P_{S} P_{R} \sigma_{e, S R}^{2}(l) \sigma_{e, R D}^{2}(l)+\left(1+\sigma_{e, R D}^{2}(l)\right) N_{0}^{2}}{\left(P_{S} \sigma_{e, S R}^{2}(l)+N_{0}\right)\left[\left(P_{R}+N_{0}\right) \sigma_{e, R D}^{2}(l)+N_{0}\right]}
\end{aligned}
$$

Note that although the numerical evaluation of the above expression of the SER is straightforward, it is not insightful in terms of its dependence on the system parameters like the pilot spacing or power allocation between the source terminal and relay terminal. To optimize the system parameters using (15) seems to be intractable. Therefore, a simple and insightful AUB of the SER is of special interest.

As derived in [14], we know that the instantaneous SNR of the output signal from the MRC detector is the sum of two terms: the first term is determined by the direct signal from the source terminal and the second term is determined by the relay signal from the relay terminal. Using the result in [14], the instantaneous SNR determined by the relay signal can be rewritten as

$$
\gamma_{2}(l)=\frac{x_{1}(l) x_{2}(l)}{x_{1}(l)+x_{2}(l)+\beta(l)}
$$

where

$$
\begin{aligned}
& x_{1}(l)=\frac{\left|\hat{h}_{R D}(l)\right|^{2}}{\alpha_{2}(l) \sigma_{\hat{h}, R D}^{2}(l)} \\
& x_{2}(l)=\frac{\left|\hat{h}_{S R}(l)\right|^{2}}{\alpha_{3}(l) \sigma_{\hat{h}, S R}^{2}(l)}
\end{aligned}
$$

From the definition of $\beta(l)$ in (15), it can be found that $\beta(l)>0$. Therefore, if we set $\beta(l)=0$ in (16), we get an upper bound of the $\gamma_{2}(l)$. With this observation, we obtain an upper bound of the SER by simply setting $\beta(l)=0$ in (15). After some manipulations, we obtain the AUB of the SER

$$
\bar{P}_{U B}=\frac{1}{L-1} \sum_{l=1}^{L-1} \frac{4 B}{K_{Q}} \alpha_{1}(l)\left[\alpha_{2}(l)+\alpha_{3}(l)\right]
$$

Note that $N_{0} / P_{R}$ tends to zero in high SNR regions. 
Therefore, the AUB of the SER can be further simplified as

$$
\bar{P}_{U B}=\frac{1}{L-1} \sum_{l=1}^{L-1} \frac{4 B}{K_{Q}} \alpha_{1}(l)\left[\alpha_{2}^{\prime}(l)+\alpha_{3}(l)\right]
$$

where

$$
\alpha_{2}^{\prime}(l)=\frac{P_{R} \sigma_{e, R D}^{2}(l)+N_{0}}{P_{R} \sigma_{\hat{h}, R D}^{2}(l)} \approx \alpha_{2}(l)
$$

As shown in Section 5, the AUB of the SER in (18) is very close to the exact SER, especially in high SNR regions.

\section{Parameter Optimization}

As can be seen from (18), the AUB of the SER is determined by the function

$$
M\left(L, N, P_{S}, P_{R}\right)=\sum_{l=1}^{L-1} \alpha_{1}(l)\left[\alpha_{2}^{\prime}(l)+\alpha_{3}(l)\right]
$$

It should be pointed out that $\alpha_{1}(l), \alpha_{2}^{\prime}(l), \alpha_{3}(l)$, for $l=1,2, \cdots, L-1$ are related to the parameters $L, N, P_{S}$, and $P_{R}$. This can be deduced from their definition in (15). As a result, we establish the relation between the AUB of the SER and the parameters which need to be optimized. Using the above metric as an optimality criterion, we can now study the parameter optimization problem of the considered system. In principle, we should optimize the four parameters $L, N, P_{S}$, and $P_{R}$ jointly to get the optimum system performance. However, the joint optimization problem is difficult to solve due to the form of the metric in (19). Therefore, we propose to optimize the parameters of the PSAM and the power allocation separately as shown below. Although this method is suboptimal, our simulation results show that this method provides a satisfactory performance.

\subsection{PSAM Parameter Optimization}

For the PSAM scheme, there exists a tradeoff between the system performance, pilot overhead, and receiver complexity. While a smaller pilot spacing $L$ leads to a better channel estimation, the overhead imposed by the pilot symbols reduces the effective SNR and transmission efficiency. A similar conflict also exists for the choice of the Wiener filter length $N$. A larger value of $N$ is required to improve the channel estimation, but this will increase the receiver complexity. Therefore, the parameters $L$ and $N$ should be accordingly chosen by taking all these factors into account. We will use the metric in (19) as the optimality criterion for determining appropriate values of $L$ and $N$. In particular, we will set $P_{S}=P_{R}=P / 2$, where $P$ is the total transmitted power, and try to minimize the metric $M\left(L, N, P_{S}, P_{R}\right)$ which characterizes (asymptotically) the performance of the considered system. Since there is no closed-form solution to this minimization problem, the suitable values of $L$ and $N$ can only be obtained by examining the metric $M\left(L, N, P_{S}, P_{R}\right)$, which is presented in the next section.

\subsection{Power Allocation Optimization}

Now, we will study the power allocation problem for the considered system. We assume that the parameters $L$, $N$ are fixed and the total transmitted power is $P=P_{S}+P_{R}$. Under these constraints, we are going to optimize $P_{S}$ and $P_{R}$ so that the SER performance of the system is minimized. Since the metric $M\left(L, N, P_{S}, P_{R}\right)$ characterizes (asymptotically) the SER performance of the considered system, we can state the power allocation problem as follows.

Problem Statement: Given positive integers $L, N$, find a pair of real numbers $P_{S}$ and $P_{R}$ such that the metric function $M\left(L, N, P_{S}, P_{R}\right)$ is minimized under the power constraint of the transmitted power which is fixed to $P$, i.e.,

$$
\left\{P_{S}, P_{R}\right\}=\arg \min _{P_{S}, P_{R}} M\left(L, N, P_{S}, P_{R}\right)
$$

Note that the derivatives of the metric $M\left(L, N, P_{S}, P_{R}\right)$ with respect to $P_{S}$ and $P_{R}$ will be expressed as the sum of several high-order polynomials. This prevents us from finding a closed-form solution for $P_{S}$ and $P_{R}$. Therefore, we propose to find the optimum power allocation by means of a gradient search over a continuous range.

\section{Numerical Results}

In this section, we will first verify by simulations the correctness of the derived expression found for the AUB of the SER. We will then present some examples illustrating the parameter optimization procedure. We consider an AF cooperative communication system with 4-QAM modulation formats using the PSAM scheme for the channel estimation. Unless stated otherwise, the following parameters are used in the numerical work. We set $P_{S}=P_{R}$ and assume that the variance of the noise was chosen to be $N_{0}=1$. We also assume that the complex channel gains are described by the autocorrelation functions $R_{S D}(\kappa)=R_{S R}(\kappa)=R_{R D}(\kappa)=J_{0}\left(2 \pi f_{\max } \kappa T_{s}\right)$, where $J_{0}(x)$ is the zeroth order Bessel function of the first kind, $f_{\max }$ is the maximum Doppler frequency, and $T_{s}$ is the symbol duration. Note that the variances of the complex channel gains are normalized to unity. We further assume that a pilot spacing of $L=6$ is used 
in the PSAM scheme and the LMMSE with $N=6$ is used for the channel estimation. Note that the power loss resulting from the pilots is accounted for all curves.

Figure 2 shows the theoretical AUB and the Monte Carlo simulation results of the SER for the AF cooperative communication system with 4-QAM. The results are presented for two different levels of the normalized maximum Doppler frequency, i.e $f_{\max } T_{s}=0.01$ and $f_{\max } T_{s}=0.05$. From Figure 2, we observe that the AUB fits very well with the simulated SER for both cases in high SNR regions.

Assuming $P_{S}=P_{R}$, Figure 3 plots the metric $M\left(L, N, P_{S}, P_{R}\right)$ as a function of the pilot spacing $L$ and the Wiener filter length $N$ at $\mathrm{SNR}=20 \mathrm{~dB}$ with a normalized maximum Doppler frequency of $f_{\max } T_{s}=0.05$. We observe that for a given $N$, the metric $M\left(L, N, P_{S}, P_{R}\right)$ decreases rapidly with $L$ for $L \leq 4$. This is because the energy spent by pilot symbols decreases rapidly with $L$ for $L \leq 4$. As a result, the energy assigned to each data symbol increases, and this leads to a fast decrease in the SER. On the other hand, we also find that the metric $M\left(L, N, P_{S}, P_{R}\right)$ increases with $L$ for $L>7$. This is easy to understand since large $L$ will increase the channel estimation error, and thus increase the SER. By taking all these factors into account, we suggest to choose $L=6$. Now let us consider the choice of $N$. From Figure 3, we observe that for a given $L$, the metric $M\left(L, N, P_{S}, P_{R}\right)$ decreases rapidly with $N$ for $N \leq 6$. However, the decrease in $M\left(L, N, P_{S}, P_{R}\right)$ obtained by increasing $N$ beyond 6 is minor. Since large $N$ leads to a high receiver complexity, we suggest to choose $N=6$ for this particular case.

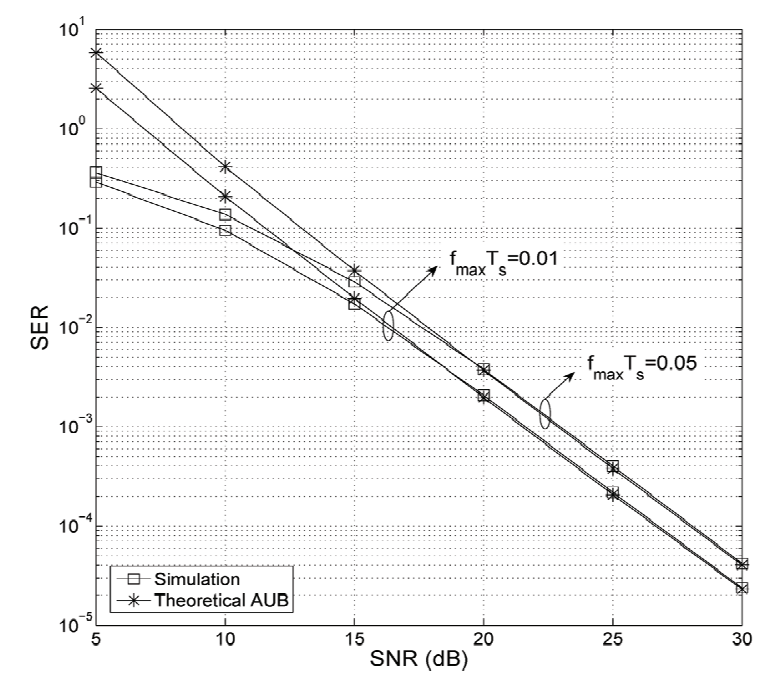

Figure 2. Comparison of the theoretical AUB and simulation results of the SER for the AF cooperative communication systems with various values of the normalized maximum Doppler frequency $f_{\max } T_{s}$.

Now, we turn our attention to the power allocation strategies. As discussed earlier, we use a constrained gradient-search algorithm to find the power tradeoff between the source terminal and the relay terminal. For example, in case of $\sigma_{S D}^{2}=\sigma_{S R}^{2}=\sigma_{R D}^{2}=1$, and $f_{\max } T_{s}=0.01$ we find the optimum power allocation is $P_{S} / P=0.66$, and $P_{R} / P=0.34$. The performance comparison of the equal power scheme and the optimum power allocation scheme is presented in Figure 4. This figure illustrates that the performance of the system with optimum power allocation is better than that of the system with equal power allocation. We can see that a greater performance improvement can be achieved from

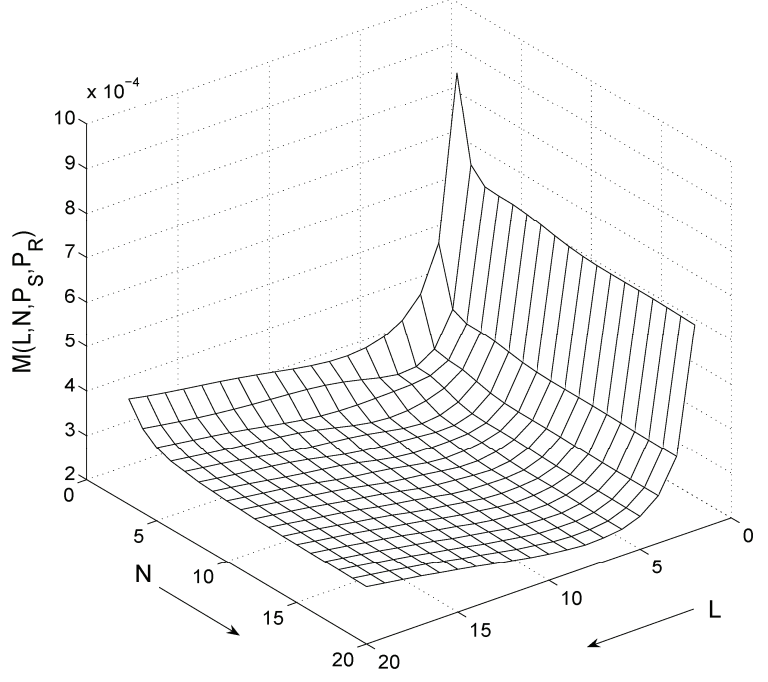

Figure 3. The metric $M\left(L, N, P_{S}, P_{R}\right)$ at $\mathrm{SNR}=20 \mathrm{~dB}$ with a normalized maximum Doppler frequency of $f_{\max } T_{s}=0.05$.

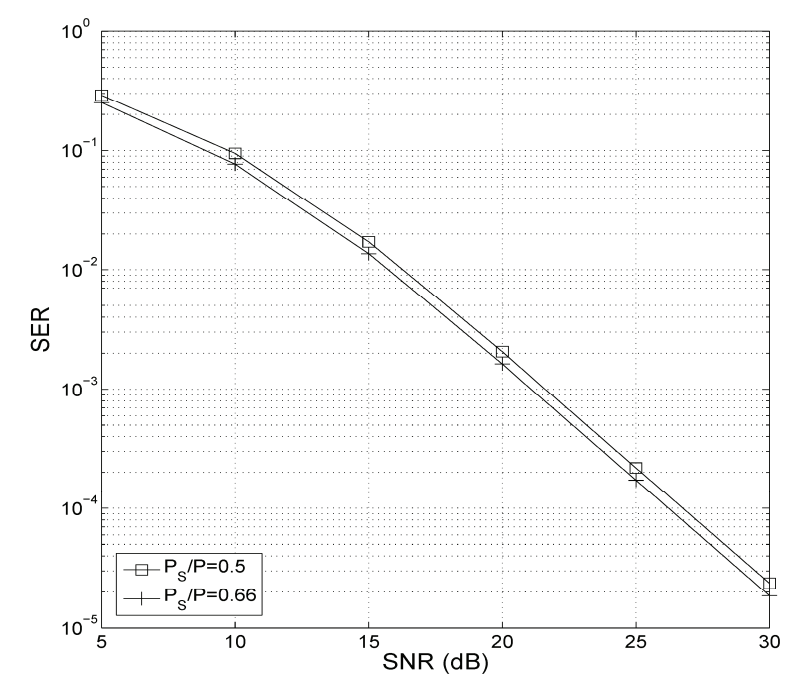

Figure 4. SER performance of the AF cooperative communication systems assuming equal power allocation and optimum power allocation. 


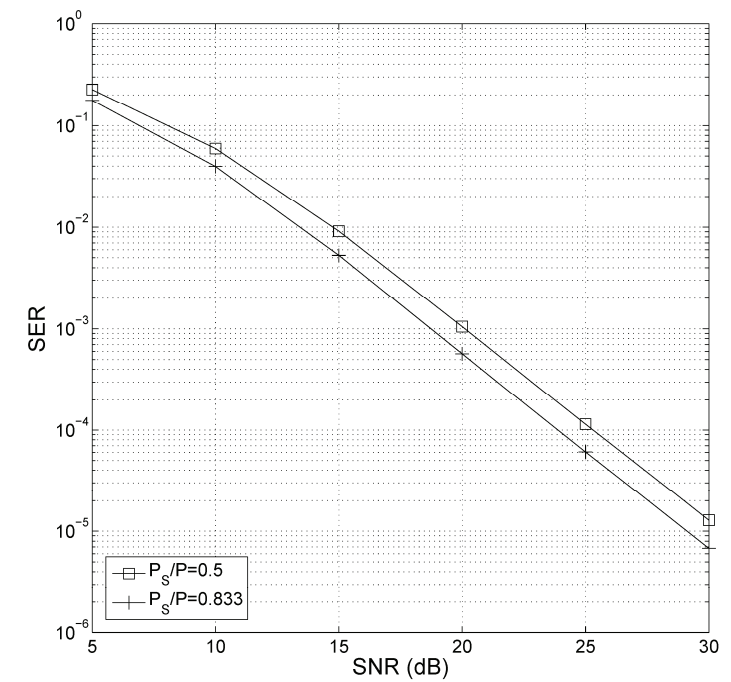

Figure 5. SER performance of the AF cooperative communication systems assuming equal power allocation and optimum power allocation.

the optimum power allocation scheme if the ratio $\sigma_{S R}^{2} / \sigma_{R D}^{2}$ decreases. For example, in case of $\sigma_{S D}^{2}=\sigma_{S R}^{2}=1, \sigma_{R D}^{2}=10$ and $f_{\max } T_{s}=0.01$, we find the optimum power allocation is $P_{S} / P=0.83$, and $P_{R} / P=0.17$. The corresponding performance comparison is plotted in Figure 5. As can be seen from this figure, the optimum power allocation scheme leads to an improvement of $1.5 \mathrm{~dB}$ over the equal power scheme. This further demonstrates the effectiveness of the power allocation optimization.

\section{Conclusions}

We dealt with the problem of parameter optimization of AF cooperative communication systems with a PSAMbased LMMSE scheme used for the channel estimation. A tight and easy to-evaluate AUB of the SER was derived for the considered system with QAM constellations. Using the derived AUB, we proposed a criterion for the choice of parameters in the PSAM scheme, i.e., pilot spacing and Wiener filter length. We also formulated an optimum power allocation problem for the considered system. The optimum power allocation was found by means of a gradient search over a continuous range. Some illustrative examples for the parameter optimization were presented. The benefits of parameter optimization were demonstrated by the numerical results.

\section{References}

[1] G. Kramer, M. Gastpar, and P. Gupta, "Cooperative strategies and capacity theorems for relay networks," IEEE Transactions on Information Theory, Vol. 51, No. 9, pp. 3037-3063, September 2005.
[2] A. Reznik, M. R. Kulkarni, and S. Verdu, "Degraded Gaussian multirelay channel: Capacity and optimal power allocation," IEEE Transactions on Information Theory, Vol. 50, No. 12, pp. 3037-3046, December 2004.

[3] A. Host-Madsen and J. Zhang, "Capacity bounds and power allocation for wireless relay channels," IEEE Transactions on Information Theory, Vol. 51, No. 6, pp. 2020-2040, June 2005.

[4] J. N. Laneman, D. N. C. Tse, and G. W. Wornell, "Cooperative diversity in wireless networks: Efficient protocols and outage behavior," IEEE Transactions on Information Theory, Vol. 50, No. 12, pp. 3062-3080, December 2004.

[5] C. S. Patel and G. L. Stüber, "Channel estimation for amplify and forward relay based cooperation diversity systems," IEEE Transactions on Wireless Communications, Vol. 6, No. 6, pp. 2348-2356, June 2007.

[6] R. U. Nabar, H. Bölcskei, and F. W. Kneubühler, "Fading relay channels: Performance limits and space-time signal design," IEEE Journal on Selected Areas in Communications, Vol. 22, No. 6, pp. 1099-1109, August 2004.

[7] M. O. Hasna and M.-S. Alouini, "Harmonic mean and end-to-end performance of transmission systems with relays," IEEE Transactions on Communications, Vol. 52, No. 1, pp. 130-135, January 2004.

[8] M. O. Hasna and M.-S. Alouini, "A performance study of dual-hop transmissions with fixed gain relays," IEEE Transactions on Wireless Communications, Vol. 3, No. 6, pp. 1963-1968, November 2004.

[9] P. A. Anghel and M. Kaveh, "Exact symbol error probability of a cooperative network in a Rayleigh-fading environment," IEEE Transactions on Wireless Communications, Vol. 3, No. 5, pp. 1416-1421, September 2004.

[10] A. Ribeiro, X. Cai, and G. B. Giannakis, "Symbol error probabilities for general cooperative links," IEEE Transactions on Wireless Communications, Vol. 4, No. 3, pp. 1264-1273, March 2005.

[11] Y. Li and S. Kishore, "Asymptotic analysis of amplifyand-forward relaying in Nakagami-fading environments," IEEE Transactions on Wireless Communications, Vol. 6, No. 12, pp. 4256-4262, December 2007.

[12] W. Su, A. K. Sadek, and K. J. R. Liu, "Cooperative communication protocols in wireless networks: Performance analysis and optimum power allocation," Wireless Personal Communications (Springer), Vol. 44, No. 2, pp. 181-217, January 2008.

[13] B. Gedik and M. Uysal, "Two channel estimation methods for amplify-and-forward relay networks," in Proceedings of Canadian Conference on Electrical and Computer Engineering , pp. 615-618, May 2008.

[14] Y. Wu and M. Pätzold, "Performance analysis of cooperative communication systems with imperfect channel estimation," IEEE International Conference on Communications, IEEE ICC 2009, accepted for publication, June 2009.

[15] L. L. Scharf, "Statistical signal processing: Detection, estimation, and time-series analysis," Reading, MA: Addison-Wesley, 1990.

[16] D. G. Brennan, "Linear diversity combining techniques," Proceedings of the IEEE, Vol. 91, No. 2, pp. 331-356, February 2003. 